\title{
A robust high-order lattice adaptive notch filter and its application to narrowband noise cancellation
}

\author{
Seong-woo Kim ${ }^{1}$, Young-cheol Park ${ }^{2}$, Young-soo Seo ${ }^{3}$ and Dae Hee Youn ${ }^{*}$
}

\begin{abstract}
In this paper, we propose a high-order lattice adaptive notch filter (LANF) that can robustly track multiple sinusoids. Unlike the conventional cascade structure, the proposed high-order LANF has robust tracking characteristics regardless of the frequencies of reference sinusoids and initial notch frequencies. The proposed high-order LANF is applied to a narrowband adaptive noise cancellation (ANC) to mitigate the effect of the broadband disturbance in the reference signal. By utilizing the gradient adaptive lattice (GAL) ANC algorithm and approximately combining it with the proposed high-order LANF, a computationally efficient narrowband ANC system is obtained. Experimental results demonstrate the robustness of the proposed high-order LANF and the effectiveness of the obtained narrowband ANC system.
\end{abstract}

Keywords: Adaptive filter; High-order lattice adaptive notch filter; Adaptive line enhancement; Narrowband adaptive noise cancellation

\section{Introduction}

Retrieving or eliminating multiple sinusoids buried in broadband noise is an important problem in many applications, such as narrowband noise cancellation [1] and active noise control [2]. In the narrowband adaptive noise cancellation (ANC) application, when the reference signal is contaminated by a broadband disturbance, the performance of the narrowband ANC system is seriously degraded. Hence, to achieve better cancellation, it is essential to improve the signal-to-noise ratio (SNR) of the reference signal comprising multiple sinusoids. A convenient way to improve the SNR of such signals at a low computational cost is to use a line enhancer based on an infinite impulse response (IIR) adaptive notch filter (ANF) [3].

The IIR ANF can be straightforwardly realized in a canonical direct form [3]. Alternative realizations have been studied in [4-6], where a number of second-order lattice adaptive notch filters (LANF) were cascaded to retrieve multiple sinusoids. However, as indicated in [6],

*Correspondence: dhyoun@yonsei.ac.kr

${ }^{1}$ School of Electrical and Electronic Engineering, Yonsei University,

Shinchondong, Seodaemoon-gu, Seoul 120-749, South Korea

Full list of author information is available at the end of the article the frequency estimates obtained with this approach are biased due to the assumption that the contraction factor is close to unity. Moreover, the tracking performance of the cascade second-order LANF is sensitive to the initial notch frequencies. To mitigate the dependency on the frequencies of the sinusoids, the complex-coefficient ANF (CANF) [7-9] was proposed. However, its tracking performance is still sensitive to the initial notch frequencies.

In this paper, we propose a high-order LANF that is not associated with any assumption for the contraction factor. By simultaneously tracking multiple sinusoids, the proposed high-order LANF is capable of robustly tracking multiple sinusoids regardless of the frequencies of the sinusoids and initial notch frequencies. Hence, the proposed high-order LANF can be applied to the narrowband ANC problem, in which the reference signal is contaminated by a broadband disturbance.

The most commonly used adaptive algorithm for the narrowband ANC system is the least mean square (LMS) algorithm, which has low computational complexity and robust performance [10]. However, the LMS algorithm has a slow convergence speed, and its cancellation performance is degraded in the presence of broadband disturbances at the reference sensor [11]. It was shown in

\section{量 Springer}

(c) 2014 Kim et al: licensee Springer. This is an Open Access article distributed under the terms of the Creative Commons Attribution License (http://creativecommons.org/licenses/by/4.0), which permits unrestricted use, distribution, and reproduction in any medium, provided the original work is properly credited. 
[11] that when the primary noise consists of multiple sinusoids, the steady-state MSE of the LMS algorithm is nearly inversely proportional to both the SNR of the reference signal and the regression filter order. Recently developed adaptive algorithms [12,13] have achieved fast convergence speed and low steady-state MSE. However, because these algorithms do not consider the presence of the broadband disturbances at the reference sensor, the degradation of the steady-state performance of the algorithms is inevitable.

In this paper, the gradient adaptive lattice (GAL) algorithm is utilized to achieve fast convergence speed for a reference signal consisting of multiple sinusoids [14-16]. In addition, the problem of the noisy reference signal is mitigated using the high-order LANF at the reference channel. However, the direct combination of the GAL narrowband ANC system with a high-order LANF has a high computational cost because the adaptive filters need to be independently updated. Thus, to obtain a computationally efficient narrowband ANC system, we present a new GAL narrowband ANC system in which only the all-pole part of the high-order LANF is used to enhance the SNR of the noisy reference signal.

This paper is organized as follows: The high-order LANF is presented in Section 2. In Section 3, the effect of the broadband disturbance in the reference signal is analyzed and the new GAL narrowband ANC system is presented. Experimental results are provided in Section 4, where the performance of the proposed high-order LANF and of the new GAL narrowband ANC system is evaluated and compared with that of the conventional algorithms. Conclusions are drawn in Section 5.

\section{High-order lattice adaptive notch filter}

The transfer function of the IIR ANF is given by [3]

$$
\frac{R(z)}{Q(z)}=\frac{1+a_{1} z^{-1}+a_{2} z^{-2}+\cdots+a_{L} z^{-L}}{1+\alpha a_{1} z^{-1}+\alpha^{2} a_{2} z^{-2}+\cdots+\alpha^{L} a_{L} z^{-L}}
$$

where $L$ is the order of the IIR ANF, and $0<\alpha<1$ is the contraction factor that determines the notch bandwidth. The IIR ANF in Equation 1 can be directly realized using the recursive prediction error algorithm [3]. In [6], a simpler ANF realization was introduced by utilizing a second-order LANF. However, the cascade second-order LANF has a biased notch response due to the assumption that the contraction factor is close to unity [6].

In this paper, we present a new high-order LANF that constructs the transfer function Equation 1 and is not associated with any assumption regarding the contraction factor. First, the all-zero part $R(z)$ of the notch filter in Equation 1 can be reconstructed using an all-zero lattice filter (lattice predictor) with order-recursive equations [10]:

$$
\begin{aligned}
& f_{l}^{r}(n)=f_{l-1}^{r}(n)+\kappa_{l}(n-1) b_{l-1}^{r}(n-1), \\
& b_{l}^{r}(n)=b_{l-1}^{r}(n-1)+\kappa_{l}(n-1) f_{l-1}^{r}(n),
\end{aligned}
$$

where $f_{l}^{r}(n)$ and $b_{l}^{r}(n)$ denote the $l$ th stage forward and backward prediction errors, respectively, and $\kappa_{l}(n)$ is the reflection coefficient of the $l$ th stage. It is straightforward to relate the coefficients $\left\{a_{l}\right\}$ in Equation 1 to the reflection coefficients $\left\{k_{l}\right\}[10]$.

Similarly, the all-pole part $1 / Q(z)$ of the notch filter can also be reconstructed using an all-pole lattice filter. To this end, we first rearrange the terms in Equation 2, as [10]

$$
f_{l-1}^{r}(n)=f_{l}^{r}(n)-\kappa_{l}(n-1) b_{l-1}^{r}(n-1),
$$

where the forward prediction error $f_{l}(n)$ is now treated as an input variable for the $l$ th stage of the rewired lattice filter. However, Equation 3 is left intact. Equations 4 and 3 define the input-output relations of the $l$ th stage in the rewired lattice filter. Next, reordering the forward and backward prediction errors as $f_{l^{\prime}}^{q}(n)=f_{l-1}^{r}(n)$, and $b_{l^{\prime}}^{q}(n)=b_{l-1}^{r}(n)$, and weighting the past backward prediction error $b_{l^{\prime}}^{q}(n)$ with the contraction factor $\alpha$, we can specify the all-pole part of the notch filter $1 / Q(z)$ :

$$
\begin{gathered}
f_{l^{\prime}}^{q}(n)=f_{l^{\prime}-1}^{q}(n)-\alpha \kappa_{L-l^{\prime}+1}(n-1) b_{l^{\prime}}^{q}(n-1), \\
b_{l^{\prime}-1}^{q}(n)=\alpha b_{l^{\prime}}^{q}(n-1)+\kappa_{L-l^{\prime}+1}(n-1) f_{l^{\prime}}^{q}(n),
\end{gathered}
$$

where $f_{l^{\prime}}^{q}(n)$ and $b_{l^{\prime}}^{q}(n)$ denote the $l^{\prime}$ th stage forward and backward prediction errors of the all-pole lattice filter, respectively. The notch filter in Equation 1 can now be implemented by cascading the all-pole and all-zero lattice filters, which are shown in Figure 1. Thus, inputs of the lattice filters are given by $f_{0}^{q}(n)=x(n)$ and $f_{0}^{r}(n)=$ $b_{0}^{r}(n)=x_{q}(n)$, where $x_{q}(n)$ denotes the output of the all-pole lattice filter.

Similarly to the direct form IIR ANF [3], adaptation of the high-order LANF can be performed by updating the reflection coefficients $\left\{\kappa_{l}\right\}$ in the all-zero lattice filter and copying the updated reflection coefficients to the all-pole lattice filter. For the adaptive implementation, the $l$ th stage reflection coefficient can be updated using the following equation [10]:

$$
\begin{aligned}
\kappa_{l}(n)= & \kappa_{l}(n-1)-\frac{\mu}{\xi_{l-1}(n)} \\
& \times\left[f_{l}^{r}(n) b_{l-1}^{r}(n-1)+f_{l-1}^{r}(n) b_{l}^{r}(n)\right],
\end{aligned}
$$

where

$$
\begin{aligned}
\xi_{l}(n) & =\beta \xi_{l}(n-1)+(1-\beta)\left[b_{l}^{r 2}(n-1)+f_{l}^{r 2}(n)\right], \\
0 & <\beta<1
\end{aligned}
$$




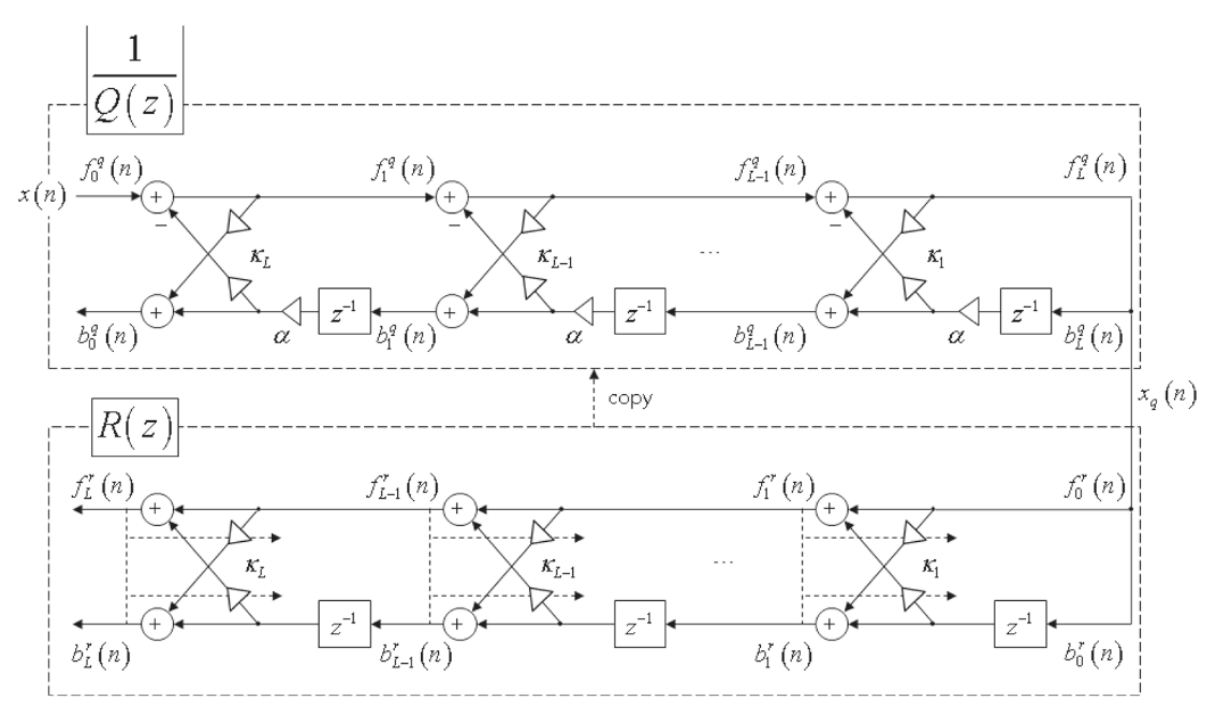

Figure 1 Proposed high-order lattice adaptive notch filter.

is an estimate of the power of both the lth stage forward and the delayed backward prediction errors of the allzero lattice filter, and $\beta$ denotes the smoothing factor. The performance analysis of Equation 7 is well derived in [16].

To estimate the frequencies of multiple sinusoids, the reflection coefficients of the proposed high-order LANF need to be transformed into the coefficients of the canonical direct form in Equation 1 via the Levinson-Durbin algorithm. The frequencies of the sinusoids can then be recursively computed from the roots of the high-order polynomial, which can be computationally costly. However, in applications such as line enhancement for the narrowband ANC system, explicit computation of the frequencies of the reference sinusoids is not required; an accurate and fast retrieval of the reference sinusoids is more important. Thus, in this paper, the proposed highorder LANF is applied to a narrowband ANC system in which the reference signal is contaminated by a broadband disturbance.

\section{Narrowband ANC System}

\subsection{Effect of uncorrelated broadband disturbance in LMS} narrowband ANC system

Figure 2 shows a block diagram of the narrowband ANC system in which the reference and desired signals are contaminated by broadband disturbances $v_{r}(n)$ and $v_{p}(n)$, respectively. By assuming that the broadband disturbances, $v_{r}(n)$ and $v_{p}(n)$ in Figure 2, represent white Gaussian noise, the steady-state MSE of the LMS narrowband ANC system can be expressed as (Eq. (53) in [11]):

$$
\begin{aligned}
E\left[e^{2}(n)\right]= & \sum_{i=1}^{I} \frac{\sigma_{d_{i}}^{2}}{\left(1+M \xi_{i} / 2\right)^{2}} \\
& +\sum_{i=1}^{I} \frac{M \xi_{i} \sigma_{d_{i}}^{2} / 2}{\left(1+M \xi_{i} / 2\right)^{2}}+\sigma_{v_{p}}^{2},
\end{aligned}
$$

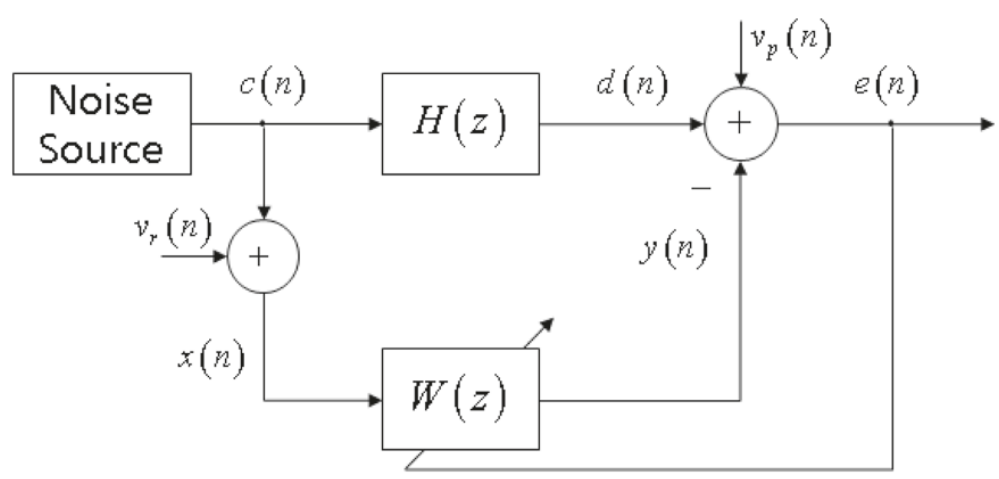

Figure 2 Conventional narrowband ANC system. 
where $\xi_{i}=\sigma_{c_{i}}^{2} / \sigma_{v_{r}}^{2}$ denotes the SNR of the reference signal. $\sigma_{c_{i}}^{2}=E\left[c_{i}^{2}(n)\right], \sigma_{d_{i}}^{2}=E\left[d_{i}^{2}(n)\right]$, and $\sigma_{v_{r}}^{2}=E\left[v_{r}^{2}(n)\right]$, $\sigma_{v_{p}}^{2}=E\left[v_{p}^{2}(n)\right]$ are the powers of the reference and primary sinusoids, and the broadband disturbances in the reference and primary channels, respectively. $I$ is the number of the sinusoids and $M$ is the order of the regression filter. The first and second terms on the right-hand side of Equation 9 represent the powers of uncanceled sinusoids and residual noise, respectively. Those terms are inversely proportional to both the regression filter order $M$ and the SNR of the reference signal. Thus, to obtain low steady-state MSE, the reference signal should maintain a high SNR condition and/or the regression filter order should be sufficiently large. However, experimental results in Section 4.2 indicate that the regression filter order needs to be several tens of times higher than necessary to suppress the effect of the broadband disturbance in the reference signal, which can be computationally too costly.

Thus, to alleviate the effect of the broadband disturbance in the reference signal, we consider a method of enhancing SNR using the proposed high-order LANF.
That is, the high-order LANF is structurally combined with the GAL narrowband ANC system and the redundant all-zero lattice filter is removed, resulting in a computationally efficient GAL narrowband ANC system.

\subsection{Robust GAL narrowband ANC system}

The LMS narrowband ANC system suffers from slow convergence, especially when the frequencies of the sinusoids in the reference signal are closely spaced [17]. The GAL narrowband ANC system can be used to overcome this problem $[14,15]$. Also, to mitigate the effect of the broadband disturbance in the reference signal, the GAL narrowband ANC system can be combined with the highorder LANF proposed in the previous section. Figure 3 shows the GAL narrowband ANC system with the ALE. The proposed high-order LANF tracks multiple sinusoids in the noisy reference signal, and multiple sinusoids are retrieved by subtracting the proposed high-order LANF output from the noisy reference signal [10]. Consequently, the ALE output $x_{o}(n)$ is expected to have a higher SNR than the noisy reference signal $x(n)$. In the GAL narrowband ANC system with the ALE, the backward prediction errors $b_{m}(n)$ are obtained from the ALE output $x_{o}(n)$.

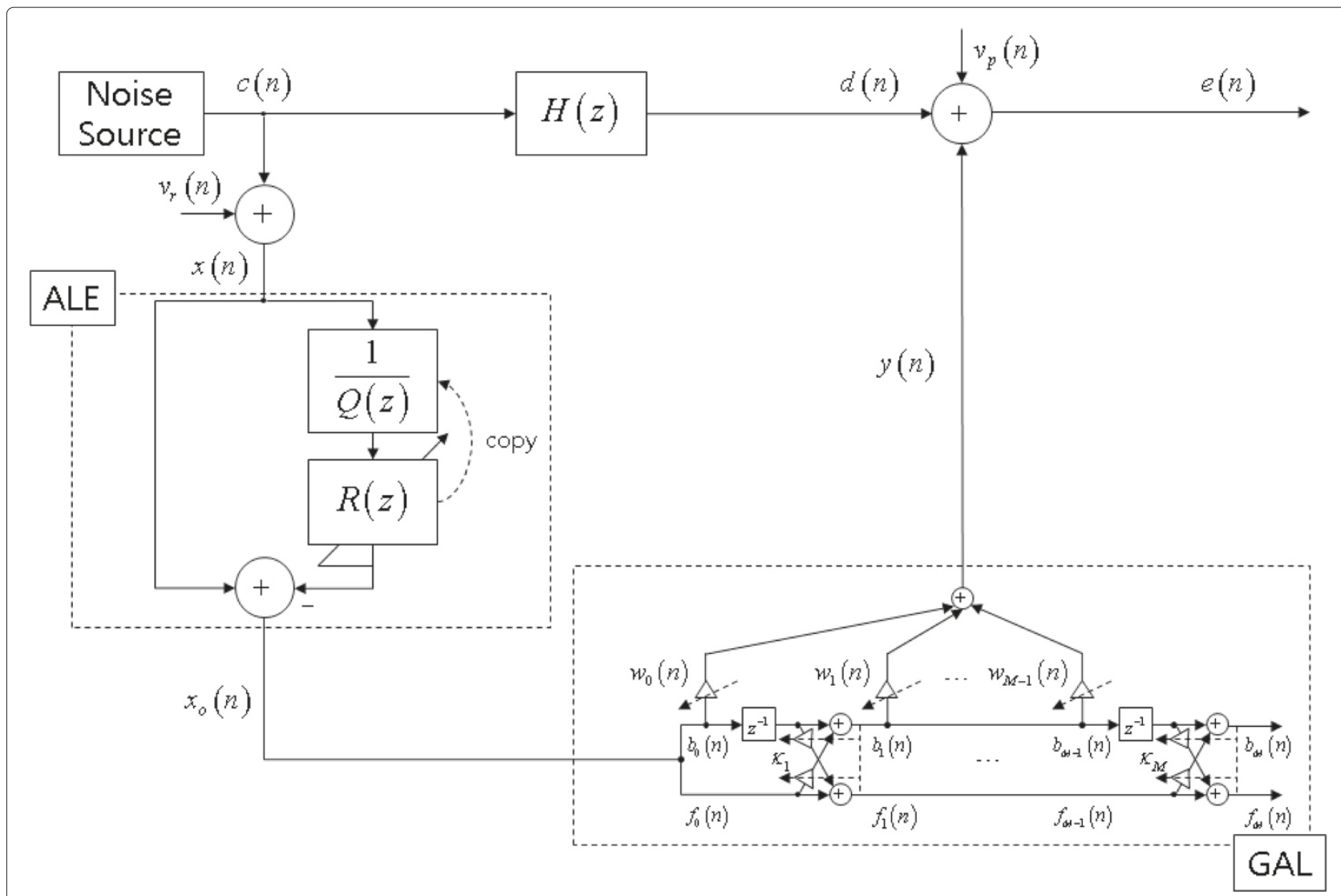

Figure $3 \mathrm{GAL}$ narrowband ANC system with ALE. 
The regression filter coefficients are then updated as follows [14]:

$$
\begin{aligned}
e_{0}(n) & =d(n), \\
e_{m}(n) & =e_{m-1}(n)+w_{m}(n-1) b_{m}(n), \\
w_{m}(n) & =w_{m}(n-1)+\frac{\mu}{\sigma_{b_{m}}^{2}(n)} b_{m}(n) e_{m}(n),
\end{aligned}
$$

where

$$
\sigma_{b_{m}}^{2}(n)=\beta \sigma_{b_{m}}^{2}(n-1)+(1-\beta) b_{m}^{2}(n)
$$

denotes an estimate of the power of the $m$ th backward prediction error.

If the proposed high-order LANF is used for the ALE, the GAL narrowband ANC system with the ALE in Figure 3 will comprise two lattice filters that need to be independently updated, which can cause a computational complexity problem. Figure 4a shows the structure of the ALE in Figure 3. The transfer function of the ALE can be rewritten as $1-R(z) / Q(z)=1 / Q(z)[Q(z)-R(z)]$. Thus, the ALE can be refigured as a series connection of all-pole and all-zero filters with transfer functions $1 / Q(z)$ and $Q(z)-R(z)$, respectively. Assuming that the reflection coefficients of $1 / Q(z)$ are obtained from $R(z)$, which performs the forward and backward linear predictions of the reference signal $x(n)$ comprising several sinusoids, the responses of the all-pole filter $1 / Q(z)$ at the frequencies of the sinusoids become much larger than unity. The allzero filter $Q(z)-R(z)$ then normalizes the response of $1 / Q(z)$, which results in unit responses at the frequencies of the sinusoids. But the responses become less than unity except for the frequencies of the sinusoids. Thus, filtering by $Q(z)-R(z)$ can be considered a scaling process. Based on this observation, the ALE in Figure 4a can be approximated to the one in Figure 4c, where $\delta$ denotes the scaling constant that normalizes the response at the frequencies of the sinusoids. Furthermore, because the GAL narrowband ANC system performs the power normalization of the input signal, the scaling process can be omitted, which leaves only the all-pole filter $1 / Q(z)$ in the reference signal.

The remaining problem is how to estimate the reflection coefficients of the all-pole filter $1 / Q(z)$. Originally, the reflection coefficients were copied from the all-zero filter $R(z)$ [5]. To solve this problem, we can utilize the lattice predictor in the GAL cancellation filter, which estimates the reflection coefficients from the all-pole filter output $x_{q}(n)$. The reflection coefficients of the lattice predictor in the GAL cancellation filter can then be copied to the all-pole lattice filter $1 / Q(z)$.

We now have a new GAL narrowband ANC system that has fast convergence speed and is robust under the noisy reference signal condition. The proposed GAL narrowband ANC system adaptively improves the SNR of the reference signal using an all-pole lattice filter that can effectively enhance the SNR of the reference signal. A schematic diagram of the proposed GAL narrowband ANC system is shown in Figure 5. The regression filter in this system uses the all-pole lattice filter output $x_{q}(n)$ as a new reference signal. Compared to the IIR lattice-based ALE output $x_{o}(n)$ in Figure 3, the all-pole lattice filter output $x_{q}(n)$ has higher power but similar SNR. Hence, according to Equation 9, the proposed GAL narrowband ANC system can obtain a low steadystate MSE. In addition, the self-orthogonalization property of the lattice filter [14] enables improved convergence speed.

Because only the reflection coefficients of the lattice predictor in the proposed GAL narrowband ANC system are updated and because they are copied to the all-pole lattice filter $1 / Q(z)$, the increase in computational complexity compared to the conventional GAL narrowband ANC system is insignificant. In Table 1, we compare the number of multiplications required in each case of the narrowband ANC system. The proposed GAL narrowband ANC system can save more than $30 \%$ of

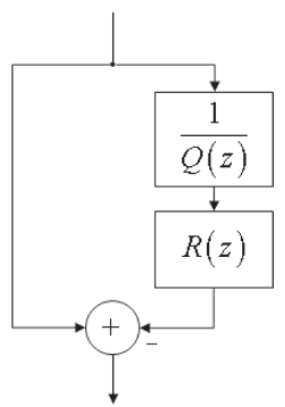

(a)

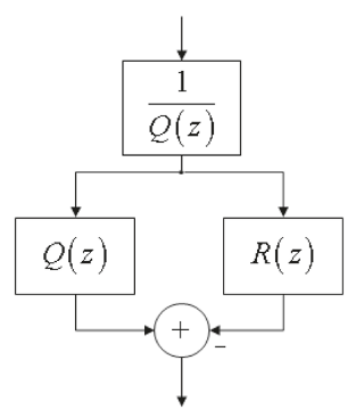

(b)

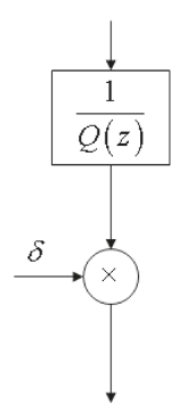

(c)

Figure 4 ALE simplification. (a) ALE; (b) equivalence of (a); (c) approximation of (b) 


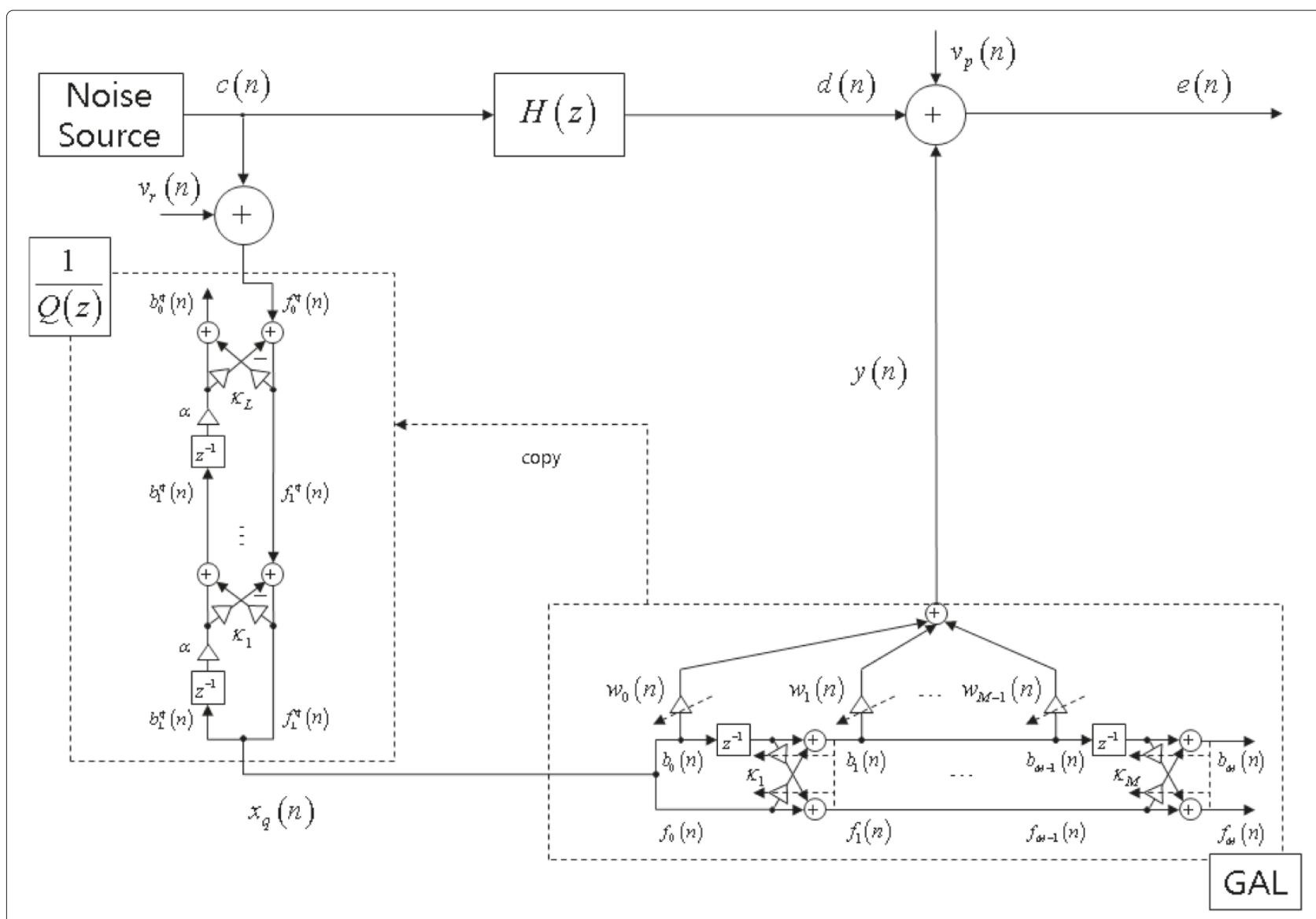

Figure 5 Proposed GAL narrowband ANC system.

the multiplications compared with the GAL narrowband ANC system directly employing an ALE. The use of the ALE increases computational complexity by several times compared with the case of not using it. However, it should be mentioned that to suppress the effect of the broadband disturbance in the reference signal without the ALE, the regression filter order of the LMS narrowband ANC system needs be much higher than the case of using the ALE. More details will be discussed in Section 4.2.

\subsection{Convergence behavior of proposed system}

If the frequencies of the sinusoids are constant, the broadband disturbance is stationary, and the step-size parameter is sufficiently small, the convergence time is dependent

Table 1 Comparison of computational complexity

\begin{tabular}{lccc}
\hline & ANC & ALE & Total \\
\hline LMS & $3 M$ & N/A & $3 M$ \\
LMS with ALE & $3 M$ & $13 M$ & $16 M$ \\
GAL with ALE & $17 M$ & $13 M$ & $30 M$ \\
Proposed & $17 M$ & $3 M$ & $20 M$ \\
\hline
\end{tabular}

on the eigenvalue spread of the reference signal correlation matrix in the LMS algorithm. When the frequencies of the sinusoids in the reference signal are closely spaced, the low bound for the eigenvalue spread of the reference signal correlation matrix increases [17]. Accordingly, the LMS narrowband ANC system suffers from a slow convergence. However, because the proposed GAL narrowband ANC system utilizes the lattice predictor, which orthogonalizes the reference signal, its convergence speed is fairly insensitive to the eigenvalue spread of the reference signal correlation matrix [14]. Hence, even if the frequencies of the sinusoids in the reference signal are closely spaced, the proposed GAL narrowband ANC system will have fast convergence speed.

According to the analysis in [3], the asymptotic SNR gain obtainable using the IIR lattice-based ALE is inversely proportional to the notch bandwidth:

$$
\frac{\mathrm{SNR}_{\mathrm{out}}}{\mathrm{SNR}_{\mathrm{in}}}=\frac{1}{1-\alpha},
$$

where $\mathrm{SNR}_{\text {in }}$ and $\mathrm{SNR}_{\text {out }}$, respectively, denote the input and output SNRs of the ALE. Because the all-zero part of the ALE $Q(z)-R(z)$ in Figure 4b can be approximated 
as a scaling process, the all-pole lattice filter $1 / Q(z)$ is expected to have an SNR gain similar to that of the ALE, which will be verified with experimental results in Section 4.

In ([11] Eq. (53)), the correlation matrix of the ALE output is used to obtain the steady-state MSE, but the closed-form expression of the steady-state MSE will be very complicated, because the ALE output is no longer white Gaussian noise [11]. However, neglecting the offdiagonal elements of the correlation matrix and using only the diagonal elements $(1-\alpha) \sigma_{v_{r}}^{2}$ of the correlation matrix, the steady-state MSE of the proposed GAL narrowband ANC system can be approximated as

$$
\begin{aligned}
E\left[e^{2}(n)\right]= & \sum_{i=1}^{I} \frac{\sigma_{d_{i}}^{2}}{\left(1+M \xi_{i} / 2(1-\alpha)\right)^{2}} \\
& +\sum_{i=1}^{I} \frac{M \xi_{i} \sigma_{d_{i}}^{2} / 2(1-\alpha)}{\left(1+M \xi_{i} / 2(1-\alpha)\right)^{2}}+\sigma_{v_{p}}^{2} .
\end{aligned}
$$

Although neglecting the off-diagonal elements can cause errors between the theoretical and simulated steady-state MSEs, Equation 15 can be considered a lower bound to the steady-state MSE of the proposed GAL narrowband ANC system, which will be brought into conformity with the simulation results in Section 4.2. Because the contraction factor $\alpha$ is smaller but closer to unity, the proposed GAL narrowband ANC system is expected to yield lower steady-state MSEs than the conventional systems.

\section{Experimental results}

In this section, we present experimental results for the proposed high-order LANF and GAL narrowband ANC system. We first compare the convergence speed and tracking ability of the high-order LANF with those of the conventional ANF in [6] and [9]. Subsequently, the performance of the GAL narrowband ANC system employing the high-order LANF is evaluated, and it is then compared with performance of adaptive filters recently proposed in [12] and [13].

\subsection{Performance evaluation of high-order LANF}

In this experiment, the tracking performance of the proposed high-order LANF is compared with that of the cascade second-order LANF [6] and that of cascade firstorder CANF [9]. Two sinusoids having unit amplitudes and random phases with uniform distribution over $[0, \pi]$ were used as the input to the ANF. The frequency of the first sinusoid was $100 \mathrm{~Hz}$, and the frequency of the second sinusoid was varied from 200 to $180 \mathrm{~Hz}$ and again to $200 \mathrm{~Hz}$ at 5 and $10 \mathrm{~s}$, respectively. White Gaussian noise was added at $0 \mathrm{~dB}$ SNR, and the sampling frequency was
$1 \mathrm{kHz}$. The filter order $L$ of the ANF was 4 . The stepsize parameters were experimentally selected to achieve the best tracking performance: $\mu=0.01$ for the cascade second-order LANF, $\mu=0.0008$ for the cascade firstorder CANF, and $\mu=0.01$ for the proposed high-order LANF. The smoothing factor $\beta$ was set to $1-\mu$. The contraction factor $\alpha$ was 0.96 .

The frequencies of the sinusoids estimated by the ANFs are shown in Figure 6, where the frequencies were computed from the ANF coefficients. The results were obtained by ensemble averaging over 100 independent trials. To assess the dependency on the initial notch frequencies, the ANF coefficients were initialized with values corresponding to certain frequencies. When the coefficients were initialized to zeroes corresponding to double notches at $250 \mathrm{~Hz}$ (Figure 6, left column), the cascade second-order LANF [6] and cascade first-order CANF [9] could not properly identify the frequencies of the sinusoids, but when the coefficients were initialized to have notches at 75 and $225 \mathrm{~Hz}$, respectively, both algorithms accurately identified both frequencies, which agrees with the results of previous studies [6,9]. On the other hand, the proposed high-order LANF accurately identified the frequencies of the sinusoids regardless of the initial notch frequencies. This property is important in practical situations, such as when target frequencies are unknown.

When the number of sinusoids is more than 3 , it is not typical to compute the frequencies from the estimated reflection coefficients of the proposed high-order LANF. However, it is still possible to assess the accuracy of the retrieved sinusoids by measuring the MSE and transfer function.

Figure 7 compares the MSEs of the ANFs together with the minimum MSE corresponding to the background white-noise level. We used four sinusoids with unit amplitudes and phases with uniform distribution over $[0, \pi]$. White Gaussian noise was added at $10 \mathrm{~dB}$ SNR. The frequencies of the sinusoids were 100,200,300, and 400 $\mathrm{Hz}$, respectively, and they were changed to 100,170 , 300 , and $370 \mathrm{~Hz}$ at $5 \mathrm{~s}$. At $10 \mathrm{~s}$, they were changed to $150,250,350$, and $450 \mathrm{~Hz}$. The filter order $\mathrm{L}$ of the ANF was 8. The step-size parameters were experimentally selected to prevent the ANFs from divergence: $\mu=$ 0.006 for the cascade second-order LANF, $\mu=0.001$ for the cascade first-order CANF, and $\mu=0.0045$ for the proposed high-order LANF. The contraction factor $\alpha$ was 0.96 . All the coefficients of the ANFs were initialized to zeroes. The results in Figure 7 show that the previous cascade ANFs suffer from poor tracking ability, especially when there are sudden frequency changes, and their performance varies according to the input and initial notch frequencies. In particular, the CANF shows 


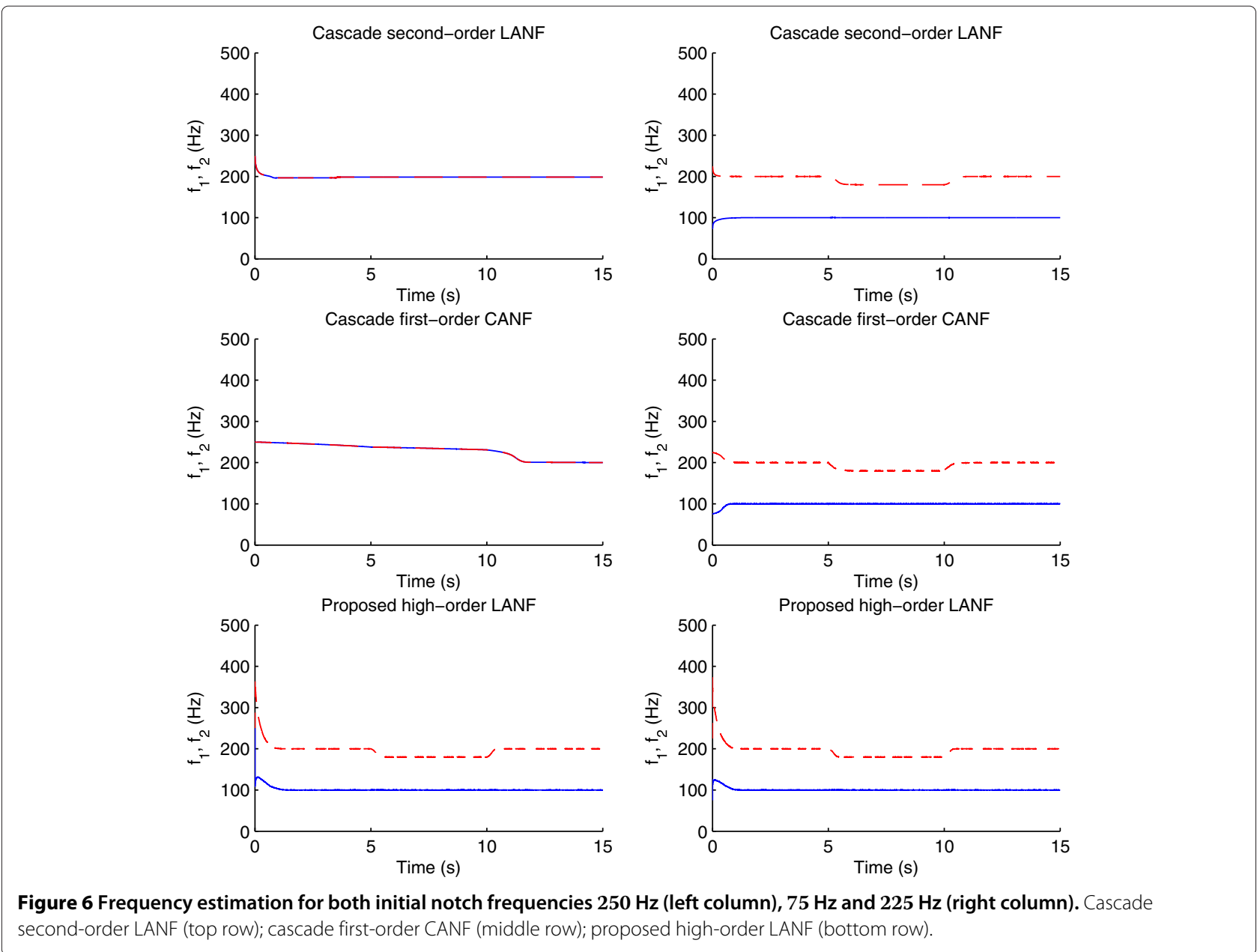

extremely slow convergence speed because its convergence speed is very sensitive to the initial notch frequencies. The proposed high-order LANF, on the other hand, shows fairly consistent convergence speed and tracking ability.

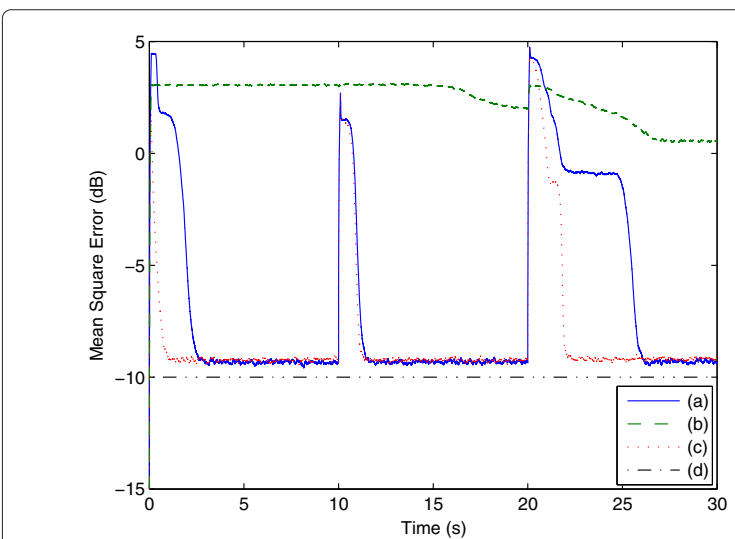

Figure 7 MSE. (a) cascade second-order LANF; (b) cascade first-order CANF; (c) proposed high-order LANF; (d) white-noise level.
More details regarding the estimated frequencies can be found from the steady-state transfer functions shown in Figure 8. The transfer functions were measured at $9 \mathrm{~s}$ (top row), $19 \mathrm{~s}$ (middle row), and $29 \mathrm{~s}$ (bottom row), respectively. 'X's mark the optimum notch frequencies. It is shown that the proposed high-order LANF places notches at exact frequencies of the reference sinusoids, whereas the transfer function of the CANF may fail to identify the reference frequencies, which results in high steady-state MSEs.

\subsection{Performance evaluation of GAL narrowband ANC}

In this experiment, we first assessed the SNR improvement by the all-pole lattice filter. We used a noise source signal comprising 200 and $250 \mathrm{~Hz}$ sinusoids with unit amplitudes. The phases of the sinusoids were uniformly distributed over $[0, \pi]$. The SNR was $20 \mathrm{~dB}$. The filter order L of the ALE was 4, the step-size parameter $\mu$ for the all-zero lattice filter (lattice predictor) was 0.001, and the contraction factor $\alpha$ was 0.99 . The power spectral densities (PSDs) of the reference input $x(n)$, the ALE output $x_{o}(n)$ in Figure 3, and the all-pole filter output 

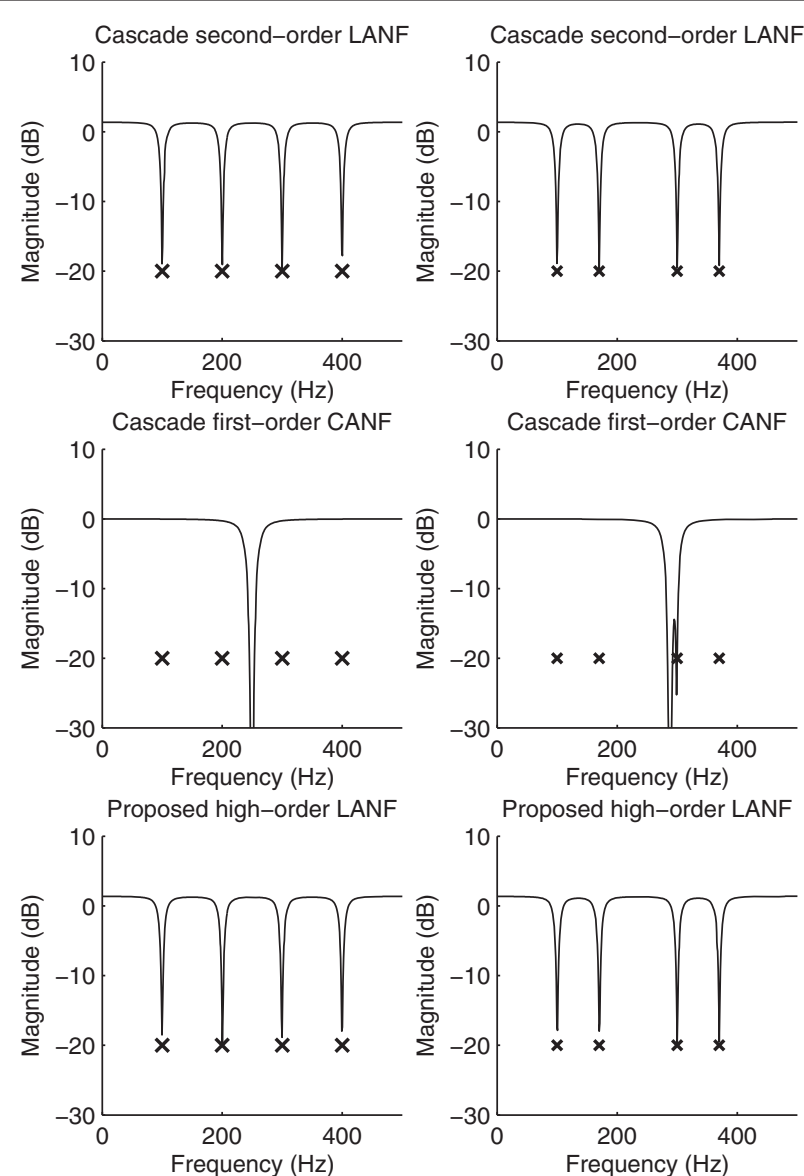
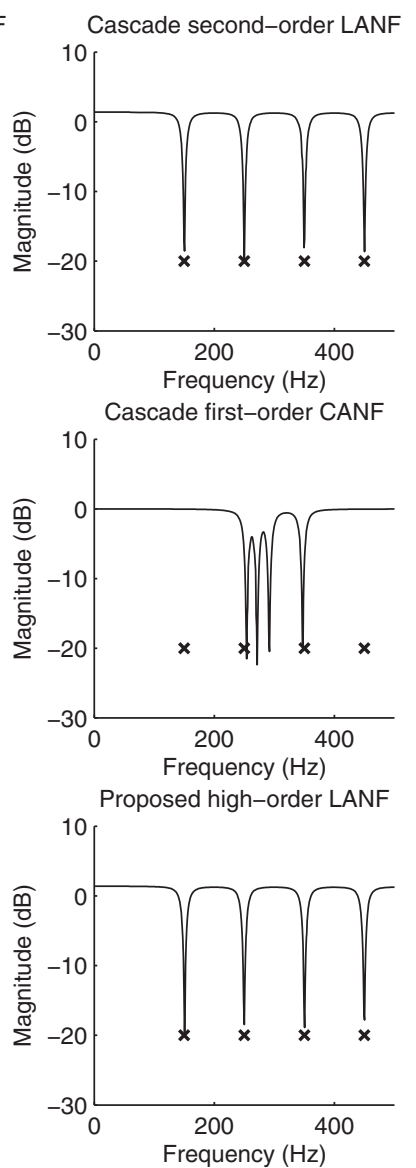

Figure 8 Transfer function for varying frequencies measured at $4 \mathrm{~s}$ (left column), $\mathbf{9} \mathrm{s}$ (middle column), $14 \mathrm{~s}$ (right column). Cascade second-order LANF (top row); cascade first-order CANF (middle row); proposed high-order LANF (bottom row).

$x_{q}(n)$ in Figure 5 are shown in Figure 9. The results show that the ALE suppresses the disturbance level at all frequencies, whereas the all-pole filter significantly amplifies the sinusoids and slightly attenuates the disturbance level. Although the all-pole filter alters the absolute level of the reference sinusoids, it should be remembered that only the SNR of the reference signal affects the steadystate MSE. Figure 9b,c clearly demonstrates that the SNR improvement of the all-pole filter is similar to that of the ALE.

Next, the narrowband ANC systems were evaluated under the noisy reference signal condition. The broadband disturbances at the reference sensor and the measurement noise at the error microphone were assumed to be white Gaussian noise. Along with the learning curves, the steady-state PSD was measured. The sampling frequency was $f_{s}=1 \mathrm{kHz}$, and the results shown below were ensemble averaged over 100 independent trials. The reference and primary signals comprising four sinusoids of frequencies 100, 200, 270, and $350 \mathrm{~Hz}$ with unit amplitudes and random phases were used. The step-size parameters were experimentally selected to equalize the steady-state MSEs of each narrowband ANC system: $\mu=0.048 / M$ for the LMS narrowband ANC system, $\mu=0.006$ for the LMS narrowband ANC system with the ALE, $\mu=$ 0.004 for the GAL narrowband ANC system with the ALE, and $\mu=0.01$ for the proposed GAL narrowband ANC system.

Figure 10 compares the convergence behaviors of the narrowband ANC systems. To confirm the tracking property, the frequencies of the sinusoids were changed to 100, 205,275 , and $350 \mathrm{~Hz}$ at $25 \mathrm{~s}$. The regression filter order $\mathrm{M}$ was 8 for all narrowband ANC systems. The order and the step-size parameter of the ALE were assumed to be the same as those of the regression filter in the narrowband ANC systems. It can be seen from the figure that the convergence speed of the proposed GAL narrowband ANC system is much faster than that of the LMS narrowband ANC system with the ALE, and that it is similar to that of the GAL narrowband ANC system with the ALE. However, as shown in Table 1, the computational complexity of the proposed GAL narrowband 

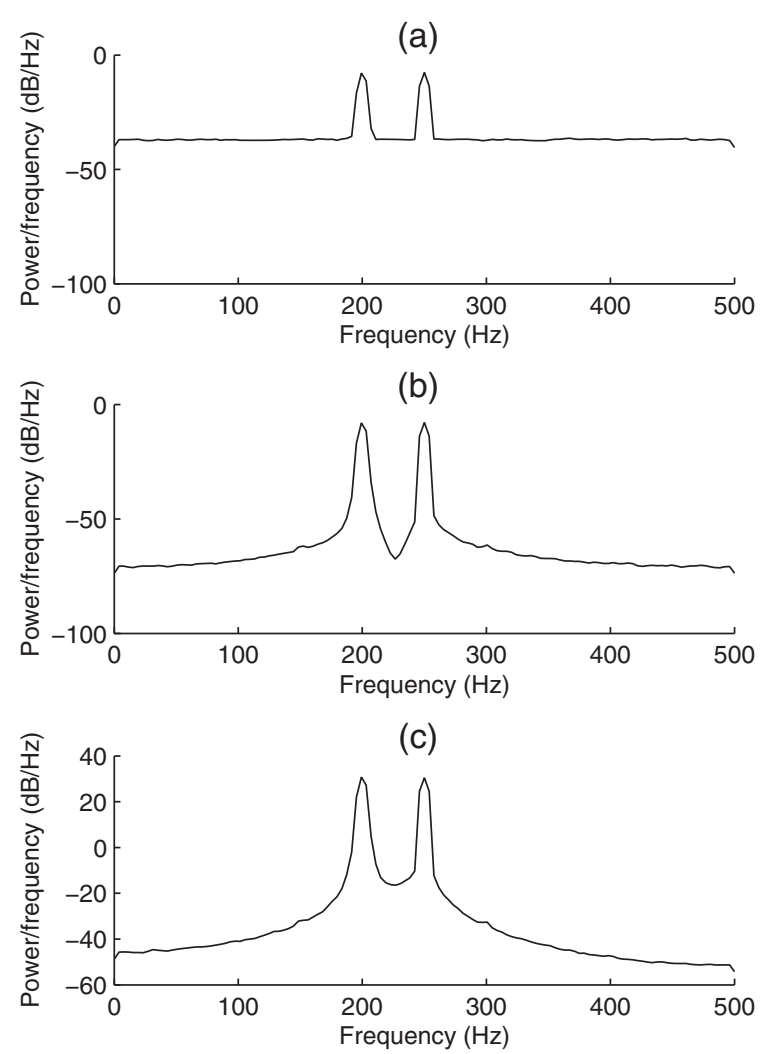

Figure 9 PSD. (a) Reference signal; (b) ALE output; (c) all-pole filter output.

ANC system is much lower than that of the GAL narrowband ANC system with the ALE. The LMS narrowband ANC system shows extremely slow convergence speed; moreover, it shows significantly higher MSE than do the others. This is because the broadband disturbance in the reference signal actually increases the steady-state MSE

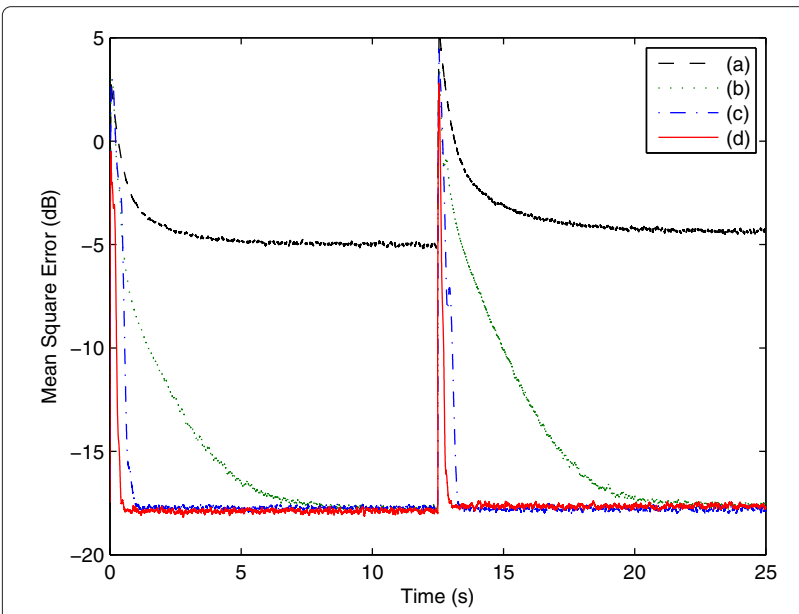

Figure 10 MSE. (a) LMS narrowband ANC system; (b) LMS narrowband ANC system with ALE; (c) GAL narrowband ANC system with $A L E ;(d)$ proposed GAL narrowband ANC system. of the LMS narrowband ANC system. The steady-state PSDs of error signals are compared in Figure 11, which clearly shows that the use of the ALE in the reference signal improves the cancellation of sinusoids and suppresses the effect of the broadband disturbances. The proposed

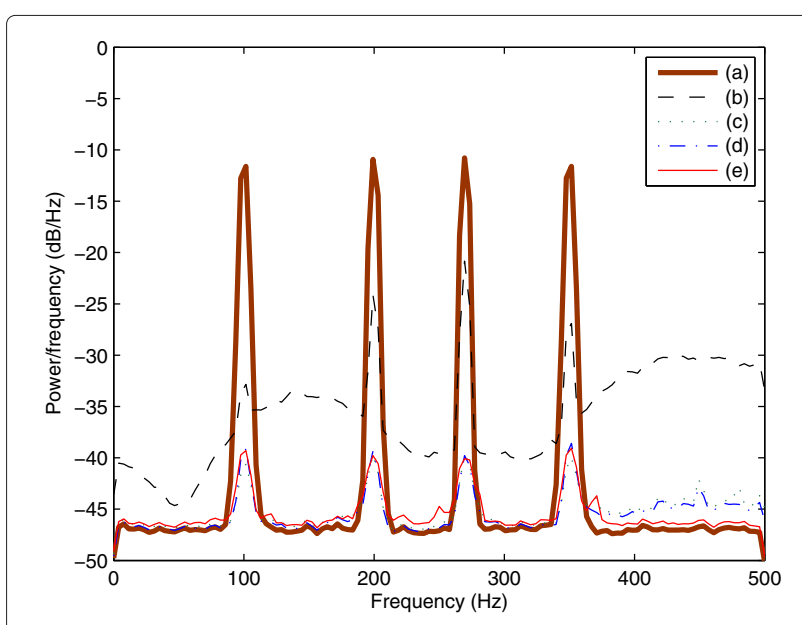

Figure 11 PSD of the error signals. (a) ANC off; ANC on using (b) LMS narrowband ANC system; (c) LMS narrowband ANC system with ALE; (d) GAL narrowband ANC system with ALE; (e) proposed GAL narrowband ANC system. 
Table 2 Steady-state MSE

\begin{tabular}{|c|c|c|c|c|c|c|}
\hline & & & LMS & & & Proposed \\
\hline Filter order $(M)$ & 8 & 16 & 32 & 64 & 128 & 8 \\
\hline Theory & -9.60 & -12.22 & -14.56 & -16.48 & -17.89 & -19.58 \\
\hline Simulation & -5.89 & -12.18 & -14.36 & -16.42 & -17.89 & -17.90 \\
\hline
\end{tabular}

GAL narrowband ANC system performs similarly to the narrowband ANC systems employing the ALE.

For further comparison, we measured steady-state MSEs, which were calculated by averaging $50 \mathrm{~s}$ of data after the algorithms reached their steady state. The power of the broadband disturbance at the reference sensor and that of the measurement noise at the error microphone were set to $\sigma_{v_{r}}^{2}=-10 \mathrm{~dB}$ and $\sigma_{v_{p}}^{2}=-20 \mathrm{~dB}$, respectively. The regression filter order of the LMS narrowband ANC system was varied from $M=8$ to $M=128$, and the regression filter order of the proposed GAL narrowband ANC system was $M=8$. Table 2 summarizes the measured steady-state MSEs and shows the theoretical MSEs calculated using Equations 9 and 15. The table shows that only the 128th-order LMS narrowband ANC system achieves a steady-state MSE similar to that of the proposed GAL narrowband ANC system employing 8th-order filters. Thus, even considering the total computational complexity in Table 1, the proposed GAL narrowband ANC system can be more efficient than the LMS narrowband ANC system.

To confirm the robustness of the proposed GAL narrowband ANC system, the steady-state MSEs were measured according to the power variation of the broadband disturbance at the reference sensor. Table 3 shows the results. We also included theoretical MSEs for comparison. The proposed GAL narrowband ANC system always obtained MSEs similar to or slightly lower than those of the narrowband ANC systems employing the ALE. The results in Table 3 also confirm that the SNR improvement obtained by using the all-pole filter $1 / Q(z)$ is similar to that obtained by using the ALE, i.e., $1-R(z) / Q(z)$.

Table 3 Steady-state MSE according to $\sigma_{v_{r}}^{2}$

\begin{tabular}{lccccc}
\hline \multirow{2}{*}{ ANC } & \multicolumn{5}{c}{$\boldsymbol{\sigma}_{\mathbf{v}_{r}}^{\mathbf{2}}(\mathbf{d B})$} \\
\cline { 2 - 6 } & $\mathbf{1 0}$ & $\mathbf{5}$ & $\mathbf{0}$ & $\mathbf{- 5}$ & $\mathbf{- 1 0}$ \\
\hline LMS (theory) & 2.24 & 0.92 & -1.70 & -5.48 & -9.78 \\
Proposed (theory) & -9.78 & -13.86 & -17.00 & -18.81 & -19.59 \\
LMS (simulation) & 3.93 & 1.96 & 0.15 & -1.57 & -3.23 \\
LMS with ALE (simulation) & -1.94 & -6.33 & -10.68 & -14.45 & -17.02 \\
GAL with ALE (simulation) & -2.07 & -6.34 & -10.68 & -14.53 & -17.23 \\
Proposed (simulation) & -2.81 & -7.97 & -12.47 & -16.01 & -18.18 \\
\hline
\end{tabular}

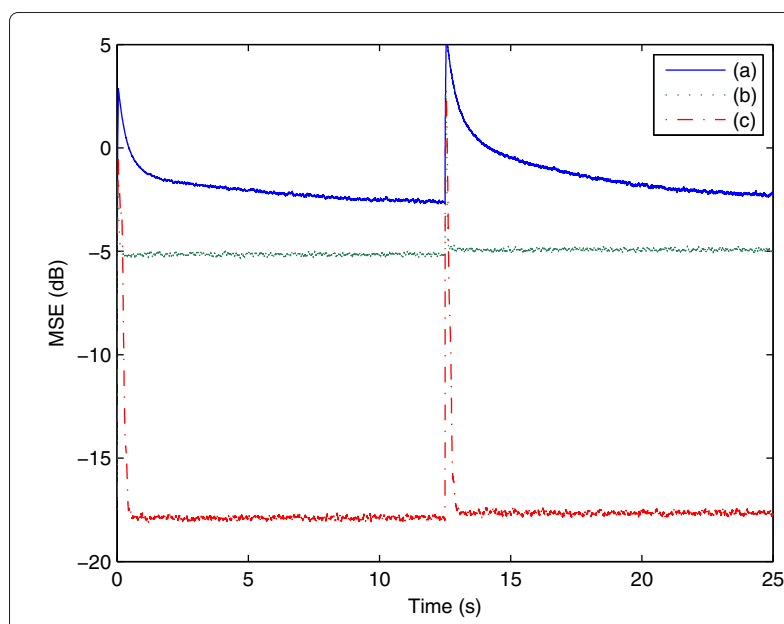

Figure 12 MSE. (a) CS-LMS narrowband ANC system; (b) VSS-GAL narrowband ANC system; (c) proposed GAL narrowband ANC system.

Finally, Figure 12 compares the convergence behavior of the proposed GAL narrowband ANC system with that of the narrowband ANC system based on recent algorithms, such as the constrained stability LMS (CS-LMS) [12] and the variable step-size gradient adaptive lattice (VSS-GAL) [13] algorithms. The step-size parameter of the CS-LMS narrowband ANC system was $\mu=0.006$. The other simulation parameters are the same as those in Figure 10. Because the previous algorithms do not consider the presence of the broadband disturbance at the reference channel, their steady-state MSEs are higher than that of the proposed GAL narrowband ANC system.

\section{Conclusions}

A new high-order LANF was presented. It was applied to the narrowband ANC system to alleviate the effect of broadband disturbances in the reference signal. By removing redundant operations, a computationally effective narrowband ANC system was obtained. Experimental results showed that the proposed high-order LANF achieved fast convergence speed and robust tracking ability regardless of the input and initial notch frequencies. In addition, the proposed narrowband ANC system achieved lower steady-state MSE and faster convergence speed than the conventional narrowband ANC algorithms.

\footnotetext{
Competing interests

The authors declare that they have no competing interests.

\section{Acknowledgements}

This work was supported by the Agency for Defense Development in the Republic of Korea (grant No. UD110034DD).

\section{Author details}

${ }^{1}$ School of Electrical and Electronic Engineering, Yonsei University, Shinchondong, Seodaemoon-gu, Seoul 120-749, South Korea. ${ }^{2}$ Computer and Telecommunications Engineering Division, Yonsei University, Wonju, Kangwon 220-710, South Korea. ${ }^{3}$ Agency for Defense Development, Changwon, Gyeongsangnam-do 645-600, South Korea.
} 


\section{References}

1. B Widrow, JR Glover JR, JM McCool, J Kaunitz, CS Williams, RH Hearn, JR Zeidler, E Dong JR, RC Goodlin, Adaptive noise cancelling: principles and applications. Proc. IEEE. 63(12), 1692-1716 (1975)

2. SJ Elliot, Signal Processing for Active Control. (Academic, London, UK, 2001)

3. A Nehorai, A minimal parameter adaptive notch filter with constrained poles and zeros. IEEE Trans. Acoust., Speech, Signal Process. 33(4), 983-996 (1985)

4. T Kwan, K Martin, Adaptive detection and enhancement of multiple sinusoids using a cascade IIR filter. IEEE Trans. Circuits Syst.,. 36(7), 937-947 (1989)

5. NI Cho, C-H Choi, SU Lee, On the adaptive lattice notch filter for the detection of sinusoids. IEEE Trans. Circuits Syst. 40(7), 405-416 (1993)

6. NI Cho, SU Lee, Tracking analysis of an adaptive lattice notch filter. IEEE Trans. Circuits Syst. 42(3), 186-195 (1995)

7. PA Regalia, A complex adaptive notch filter. IEEE Signal Process. Lett. 17(11), 937-940 (2010)

8. S Nishimura, A Mvuma, T Hinamoto, Complex adaptive notch filter for detection of real sinusoid, in Proceedings of the IEEE 54th International Midwest Symposium Circuits and Systems (MWSCAS) (Seoul, 7-10 Aug 2011)

9. S Nishimura, A Mvuma, T Hinamoto, Tracking properties of complex adaptive notch filter for detection of multiple real sinusoids, in Proceedings of the IEEE International Symposium Circuits and Systems (ISCAS) (Beijing, 19-23 May 2013)

10. S Haykin, Adaptive Filter Theory. (4th, ed.) (Prentice-Hall, Upper Saddle River, 2002)

11. NJ Bershad, JCM Bermudez, Sinusoidal interference rejection analysis of an LMS adaptive feedforward controller with a noisy periodic reference. IEEE Trans. Signal Process. 46(5), 1298-1313 (1998)

12. JM Gorriz, J Ramirez, S Cruces-Alvarez, CG Puntonet, EW Lang, D Erdogmus, A novel LMS algorithm applied to adaptive noise cancellation. IEEE Signal Process. Lett. 15(1), 34-37 (2009)

13. S-W Kim, Y-C Park, DH Youn, A variable step-size gradient adaptive lattice algorithm for multiple sinusoidal interference cancelation. EURASIP J. Adv. Signal Process (2013). doi:10.1186/1687-6180-2013-106

14. LJ Griffiths, An adaptive lattice structure for noise-cancelling applications, in Proceedings of the 1978 IEEE International Conference on Acoustics, Speech, and Signal Processing (ICASSP) (Tulsa, OK, 10-12 Apr 1978)

15. EH Satorius, JD Smith, PM Reeves, Adaptive noise cancelling of a sinusoidal interference using a lattice structure, in Proceedings of the 1979 IEEE International Conference on Acoustics, Speech, and Signal Processing (ICASSP) (Washington DC, 2-4 Apr 1979)

16. DI Kim, P Wilde, Performance analysis of the self-orthogonalising adaptive lattice filter. Signal Process. 80, 2379-2406 (2000)

17. SM Kuo, AB Puvvala, Effects of frequency separation in periodic active noise control systems. IEEE Trans. Audio Speech Lang. Process. 14(5), 1857-1866 (2006)

doi:10.1186/1687-6180-2014-137

Cite this article as: Kim et al:: A robust high-order lattice adaptive notch filter and its application to narrowband noise cancellation. EURASIP Journal on Advances in Signal Processing 2014 2014:137.

\section{Submit your manuscript to a SpringerOpen ${ }^{\mathcal{O}}$ journal and benefit from:}

- Convenient online submission

- Rigorous peer review

- Immediate publication on acceptance

- Open access: articles freely available online

- High visibility within the field

- Retaining the copyright to your article

Submit your next manuscript at $\boldsymbol{\wedge}$ springeropen.com 